novel downstream mediators of the Notch pathway in VSMC differentiation.

It is anticipated that the growing application of genomic approaches to define signature patterns in gene expression profiles during lineage commitment will lead to the discovery of new members of the vascular development gene regulatory network, advancing our understanding of human disease (21). This convergence of genomic strategies is exemplified by the recent discovery that mutations in the TGF- $\beta$ signaling pathway (a key mediator in the vascular development gene circuitry) result in a newly defined form of aortic disease (Loeys-Dietz syndrome) (22), and may foster a novel therapeutic strategy for adult vascular disease (23). Likewise, the growing integration of systems biology approaches (21) into the analysis of cardiovascular development holds promise for unlocking the remaining mysteries of the complex gene regulation circuitry governing vascular morphogenesis.

Address correspondence to: Gary H. Gibbons, 720 Westview Dr., Morehouse School of Medicine Cardiovascular Research Institute, Atlanta, Georgia 30310, USA. Phone: (404) 752-1545; Fax: (404) 752-1042; E-mail: ggibbons@msm.edu.
1. Ferguson, J.E., Kelley, R.W., and Patterson, C. 2005. Mechanisms of endothelial differentiation in embryonic vasculogenesis. Arterioscler. Thromb. Vasc. Biol. 25:2246-2254.

2. Gibbons, G.H., and Dzau, V.J. 1994. The emerging concept of vascular remodeling. New Engl. J. Med. 330:1431-1438.

3. Creazzo, T.L., Godt, R.E., Leatherbury, L., Conway, S.J., and Kirby, M.L. 1998. Role of cardiac neural crest in cardiovascular development. Annu. Rev. Physiol. 60:267-286.

4. Hirschi, K.K., and Majesky, J.W. 2004. Smooth muscle stem cells. Anat. Rec. A Discov. Mol. Cell. Evol. Biol. 276:22-33.

5. Stoller, J.Z., and Epstein, J.A. 2005. Cardiac neural crest. Semin. Cell Dev. Biol. 16:704-715.

6. High, F.A., et al. 2007. An essential role for Notch in neural crest during cardiovascular development and smooth muscle differentiation. J. Clin. Invest. 117:353-363. doi:10.1172/JCI30070.

7. Alva, J.A., and Iruela-Arispe, M.L. 2004. Notch signaling in vascular morphogenesis. Curr. Opin. Hematol. 11:278-283.

8. Xue, Y., et al. 1999. Embryonic lethality and vascular defects in mice lacking the Notch ligand Jagged1. Hum. Mol. Genet. 8:723-730.

9. Zhang, Z., Tuong, H., and Baldini, A. 2006. Mesodermal expression of Tbx 1 is necessary and sufficient for pharyngeal arch and cardiac outflow tract development. Development. 133:3587-3595.

10. Baldini, A. 2005. Dissecting contiguous gene defects: TBX1. Curr. Opin. Genet. Dev. 15:279-284.

11. Epstein, J.A., and Parmacek, M.S. 2005. Recent advances in cardiac development with therapeutic implications for adult cardiovascular disease. Circulation. 112:592-597.

12. McDaniell, R., et al. 2006. NOTCH2 mutations cause Alagille syndrome, a heterogeneous disorder of the Notch signaling pathway. Am. J. Hum. Genet. 79:169-173.
13. Campos, A.H., Wang, W., Pollman, M.J., and Gibbons, G.H. 2002. Determinants of Notch-3 receptor expression and signaling in vascular smooth muscle cells: implications in cell-cycle regulation. Circ. Res. 91:999-1006.

14. Morrow, D., et al. 2005. Cyclic strain inhibits Notch receptor signaling in vascular smooth muscle cells in vitro. Circ. Res. 96:567-575.

15. Sakata, Y., et al. 2004. Transcription factor CHF1/ Hey2 regulates neointimal formation in vivo and vascular smooth muscle proliferation and migration in vitro. Arterioscler. Thromb. Vasc. Biol. 24:2069-2074.

16. Domenga, V., et al. 2004. Notch3 is required for arterial identity and maturation of vascular smooth muscle cells. Genes Dev. 18:2730-2735.

17. Arumugam, T.V., et al. 2006. Gamma secretasemediated Notch signaling worsens brain damage and functional outcome in ischemic stroke. Nat. Med. 12:621-623.

18. Shen, H., et al. 2006. The Notch coactivator, MAML1, functions as a novel coactivator for MEF2C-mediated transcription and is required for normal myogenesis. Genes Dev. 20:675-688.

19. Doi, H., et al. 2006. Jagged1-selective Notch signaling induces smooth muscle differentiation via a RBP-J-dependent pathway. J. Biol. Chem. 281:28555-28564.

20. Proweller, A., Pear, W.S., and Parmacek, M.S. 2005. Notch signaling represses myocardin-induced smooth muscle cell differentiation. J. Biol. Chem. 280:8994-9004.

21. Olson, E.N. 2006. Gene regulatory networks in the evolution and development of the heart. Science. 313:1922-1927.

22. Loeys, B.L., et al. 2006. Aneurysm syndromes caused by mutations in the TGF- $\beta$ receptor. New Engl. J. Med. 355:788-798.

23. Habashi, J.P., et al. 2006. Losartan, an AT1 antagonist, prevents aortic aneurysm in a mouse model of Marfan syndrome. Science. 312:117-121.

\title{
An intrinsic host defense against HIV-1 integration?
}

\author{
Paul D. Bieniasz
}

Aaron Diamond AIDS Research Center and Laboratory of Retrovirology, The Rockefeller University, New York, New York, USA.

\begin{abstract}
HSCs are one of only a few cell types that resist HIV-1 infection despite the presence of HIV-1 receptors. An increasing number of genes have been identified that can reduce the sensitivity of cultured cells to retrovirus infection, and in this issue of the JCI, Zhang et al. identify $\mathrm{p}^{21^{\text {Waff }} / \operatorname{Cip} 1 / \mathrm{Sdi1}}(\mathrm{p} 21)$ as a gene product that can influence the sensitivity of HSCs to HIV-1 infection (see the related article beginning on page 473). Strikingly, p21 appears to alter the fate of nuclear HIV-1 DNA, promoting the formation of circular viral DNA forms rather than functional proviruses.
\end{abstract}

For many years, the ability of a particular retrovirus to colonize a given target cell type or species was thought to be governed solely by

Nonstandard abbreviations used: 21 , p21 Waf1/Cip1/Sdi1; PIC, HIV-1 preintegration complex.

Conflict of interest: The authors have declared that no conflict of interest exists.

Citation for this article: J. Clin. Invest. 117:302-304 (2007). doi:10.1172/JCI31290. its ability to exploit required cellular cofactors provided to it by a candidate target cell. HIV-1, for example, can only infect cells that express CD4 and a chemokine receptor because those molecules are required to mediate the fusion of virion and target cell membranes. Similarly, HIV cannot replicate in rodent fibroblasts even when they are engineered to express HIV-1 receptors because of an incompatibility between the viral and host factors required for efficient gene expression. These host cell-specific blocks have proved extremely useful in enabling researchers to infer and subsequently discover and validate the existence of host cell factors that are required for HIV-1 replication.

However, what was not appreciated until quite recently is that evolution has equipped cells with a variety of genes whose major and perhaps only role is to prevent retrovirus replication $(1,2)$. The products of these inhibitory genes, termed restriction factors, are nearly as important as required cofactors in determining the cellular host range of HIV-1 and other retroviruses. The best known and characterized of the restriction factors are encoded by the TRIMS and 
APOBEC3G genes $(3,4)$, which, based on their potency, specificity, and evident species-specific positive selection as well as the clear existence of viral countermeasures, seem likely to have evolved for the sole purpose of combating viral infection.

In addition to a number of known hostencoded antiretroviral defense mechanisms, an increasing number of genes that can reduce the sensitivity of cultured cells to retrovirus infection in a less obviously directed way have also been identified (5-9). It can be shown that the overexpression of these genes can inhibit susceptibility to retroviruses or that their underexpression can enhance susceptibility to infection. However, whether these genes directly block some critical step in the retrovirus life cycle or affect virus growth indirectly by affecting some aspect of cell physiology is not apparent. Some of these genes play important and obvious roles in the life of cells independent of retroviral infection, and so it is not clear whether their apparent antiretroviral activity is incidental.

\section{A role for $\mathrm{p} 21^{\text {Waf1/Cip } 1 / \mathrm{Sdi1}}$ in inducing HIV-1 resistance}

In this issue of the JCI (10) and in a previous study (11), Zhang et al. have identified $\mathrm{p} 21^{\text {Waf1/Cip1/Sdi1 }}(\mathrm{p} 21)$ as a gene product that can influence the sensitivity of HSCs to HIV-1 infection. HSCs are one of a few cell types, others being undifferentiated monocytes and unstimulated $\mathrm{CD}^{+}$ $\mathrm{T}$ cells, that resist HIV-1 infection despite the presence of HIV-1 receptors (12-14). In the case of HSCs, CD4 expression appears low, and previous work indicates a major block to HIV-1 infection of HSCs is at virus entry, since infection can be achieved using pseudotyped HIV-1 virions that carry a different viral envelope (14). However, later work also suggests that a second block to HIV-1 replication is imposed by p 21 because its depletion could enhance sensitivity to pseudotyped HIV-1 vectors by about 2- to 4 -fold (11). The new work in this issue extends this finding and shows that low-level-spreading HIV-1 replication can be obtained in HSCs if these cells are transfected with siRNAs that deplete p21. Additionally, Zhang et al. present a number of experiments designed to illuminate the molecular mechanism underlying the enhancement of HIV-1 replication in HSCs upon p21 depletion (10).

So, is the effect of p21 on HIV-1 infection a direct effect on the incoming virus or a secondary effect of modulating cell physi- ology? The normal cellular functions of the p 21 protein suggest that either could be the case. Notably, p21 is a cyclin-dependent kinase (CDK) inhibitor (15) and particularly targets CDKs that are active in the G1 phase of the cell cycle. In doing so, p21 inhibits cell-cycle progression in some cellular types, including HSCs (16). Although cell-cycle progression is not absolutely required for HIV-1 infection, quiescent cells (in G0 phase) are known to be very poor targets for HIV-1 infection, at least in part because reverse transcription is not completed (17). Additionally, p21 binds to factors involved in DNA repair pathways (18). The requirement for DNA repair activities in HIV-1 infection, particularly at integration, has been debated for some years, and recent findings suggest that some DNA repair activities may actually inhibit HIV-1 infection, perhaps by targeting viral DNA prior to integration into the target cell genome (19). Overall, therefore, there are at least 2 plausible models by which p21 could inhibit HIV-1 infection.

Zhang et al. (10) argue for a direct effect of p21 on incoming HIV-1. Data in the study hint that $\mathrm{p} 21$ binds to an incoming subviral complex containing HIV-1 matrix and integrase, known as the HIV-1 preintegration complex (PIC). This is consistent with the notion that the effect could be a direct one on viral components. However, caution is warranted here because this type of experiment is notoriously difficult and artifact prone. Nonetheless, it appears that the effect of p21 depletion is specific to HIV-1 infection, as low-level replication in HSCs of the simian immunodeficiency virus SIVmac251 is unaffected by p 21 depletion. The lack of effect on a related virus is more difficult, albeit not impossible, to reconcile with a model in which the effect of $\mathrm{p} 21$ depletion on HIV-1 replication has an indirect effect on cell physiology. It would be useful to know whether SIVmac251 PICs associate with p21 and what viral determinants govern the apparently discordant effects of p 21 on HIV versus SIVmac251 replication - this would provide strong clues as to an underlying mechanism. If the effects of p 21 are direct, it might be possible to show that resistance can be induced in other cellular contexts by ectopic expression of p21. For now, the central role of $\mathrm{p} 21$ in cell physiology, and likely a complex cascade of events that accompany its depletion, urge circumspection in formulating models to explain why it appears to inhibit HIV-1 replication. The authors do show that p21-depleted cells do not begin to proliferate until some time after siRNA transfection, but this does not compellingly refute the possibility that $\mathrm{p} 21$ 's effects on HIV-1 infection are indirect because the physiological changes that lead to DNA synthesis and cell division obviously precede the events themselves.

\section{Effects of p21 on HIV-1 integration}

These caveats aside, the most striking and provocative finding in the current study (10), and one that suggests a specific effect on the incoming HIV-1 PIC, is the rather dramatic difference in the fate of nascent viral DNA that was observed in HSCs upon p21 depletion. In fact, the effects of p21 depletion on the fate of HIV-1 DNA appear much greater than the effects of p21 depletion on infection (as measured by the expression of a reporter gene embedded in the HIV genome). Specifically, control-infected HSCs appear to accumulate circular forms of HIV-1 DNA while infection of p21-depleted cells results in far more integrated proviruses and very low-level circle formation. Excessive circle formation is a hallmark of failed integration; pharmacological or mutational inhibition of HIV-1 integrase catalytic activity induces precisely this phenotype. Reasonably, the authors speculate that DNA repair systems that interact with p21 (18) might be responsible for this phenomenon. The fate of HIV-1 DNA following its entry into the nucleus is a step in the viral life cycle that is increasingly recognized to be influenced by host factors; for example, LEDGF, a chromatin-associated host protein that directly binds HIV-1 integrase, appears to be rather important for integration (20). DNA repair pathways are also increasingly recognized as inhibiting retroviral infection (19); thus, there are a number of potential mechanisms by which p21 depletion could promote integration at the expense of circle formation.

\section{Conclusions}

Based on the current findings (10), it would seem premature to dub p21 a bona fide restriction factor. Nonetheless, it does join the growing list of gene products that can influence cellular sensitivity to HIV-1 infection, and its effects on nascent HIV-1 DNA are unique and interesting. Further work will be required to determine precisely how p21's effects on HIV-1 DNA are mediated and how $\mathrm{p} 21$ 's effects relate to the function 
of other host proteins that determine the fate of HIV-1 DNA once it has entered the target cell nucleus.

\section{Acknowledgments}

Work in the author's laboratory is supported by the NIH, amFAR, and the Elizabeth Glaser Pediatric AIDS Foundation.

Address correspondence to: Paul D. Bieniasz, Aaron Diamond AIDS Research Center, 455 First Avenue, New York, New York 10016, USA. Phone: (212) 448-5070; Fax: (212) 7251126; E-mail: pbienias@adarc.org.

1. Bieniasz, P.D. 2004. Intrinsic immunity: a frontline defense against viral attack. Nat. Immunol. 5:1109-1115.

2. Goff, S.P. 2004. Genetic control of retrovirus susceptibility in mammalian cells. Annu. Rev. Genet. 38:61-85.

3. Stremlau, M., et al. 2004. The cytoplasmic body component TRIM5alpha restricts HIV-1 infection in Old World monkeys. Nature. 427:848-853.

4. Sheehy, A.M., Gaddis, N.C., Choi, J.D., and Malim,
M.H. 2002. Isolation of a human gene that inhibits HIV-1 infection and is suppressed by the viral Vif protein. Nature. 418:646-650.

5. Naghavi, M.H., et al. 2006. Moesin regulates stable microtubule formation and limits retroviral infection in cultured cells. EMBOJ. 26:41-52.

6. Naghavi, M.H., Hatziioannou, T., Gao, G., and Goff, S.P. 2005. Overexpression of fasciculation and elongation protein zeta-1 (FEZ1) induces a post-entry block to retroviruses in cultured cells. Genes Dev. 19:1105-1115.

7. Ganesh, L., et al. 2003. The gene product Murr1 restricts HIV-1 replication in resting CD4+ lymphocytes. Nature. 426:853-857.

8. Boulanger, M.C., et al. 2005. Methylation of Tat by PRMT6 regulates human immunodeficiency virus type 1 gene expression. J. Virol. 79:124-131.

9. Turelli, P., et al. 2001. Cytoplasmic recruitment of INI1 and PML on incoming HIV preintegration complexes: interference with early steps of viral replication. Mol. Cell. 7:1245-1254.

10. Zhang,J., Scadden, D.T., and Crumpacker, C.S. 2007. Primitive hematopoietic cells resist HIV-1 infec-

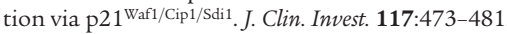
doi:10.1172/JCI28971.

11. Zhang, J., Attar, E., Cohen, K., Crumpacker, C., and Scadden, D. 2005. Silencing p21(Waf1/Cip1/Sdi1) expression increases gene transduction efficiency in primitive human hematopoietic cells. Gene Ther.
12:1444-1452.

12. von Laer, D., et al. 1990. CD34+ hematopoietic progenitor cells are not a major reservoir of the human immunodeficiency virus. Blood. 76:1281-1286.

13. Weichold, F.F., et al. 1998. Neither human immunodeficiency virus-1 (HIV-1) nor HIV-2 infects most-primitive human hematopoietic stem cells as assessed in long-term bone marrow cultures. Blood. 91:907-915.

14. Shen, H., et al. 1999. Intrinsic human immunodeficiency virus type 1 resistance of hematopoietic stem cells despite coreceptor expression. J. Virol. 73:728-737.

15. Xiong, Y., et al. 1993. p21 is a universal inhibitor of cyclin kinases. Nature. 366:701-704.

16. Cheng, T., et al. 2000. Hematopoietic stem cell quiescence maintained by $\mathrm{p} 21 \mathrm{cip} 1 / \mathrm{waf} 1$. Science. 287:1804-1808

17. Zack, J.A., et al. 1990. HIV-1 entry into quiescent primary lymphocytes: molecular analysis reveals a labile, latent viral structure. Cell. 61:213-222.

18. Waga, S., Hannon, G.J., Beach, D., and Stillman, B. 1994. The p21 inhibitor of cyclin-dependent kinases controls DNA replication by interaction with PCNA. Nature. 369:574-578.

19. Lloyd, A.G., et al. 2006. Effect of DNA repair protein Rad18 on viral infection. PLoS Pathog. 2:e40.

20. Llano, M., et al. 2006. An essential role for LEDGF/ p75 in HIV integration. Science. 314:461-464.

\title{
Transcriptional regulation of epithelial- mesenchymal transition
}

\author{
Yingqi Teng, ${ }^{1}$ Michael Zeisberg, ${ }^{1}$ and Raghu Kalluri ${ }^{1,2,3}$
}

\begin{abstract}
'Division of Matrix Biology, Beth Israel Deaconess Medical Center and Harvard Medical School, Boston, Massachusetts, USA.
2Department of Biological Chemistry and Molecular Pharmacology, Harvard Medical School, Boston, Massachusetts, USA.

${ }^{3}$ Harvard-MIT Division of Health Sciences and Technology, Boston, Massachusetts, USA.
\end{abstract}

\begin{abstract}
It has become increasingly obvious that the notion of a terminally differentiated cell is likely a simplified concept. Epithelial-mesenchymal transition (EMT), during which epithelial cells assume a mesenchymal phenotype, is a key event occurring during normal development and pathological processes. Multiple extracellular stimuli and transcriptional regulators can trigger EMT, but how such distinct signaling pathways orchestrate the complex cellular events that facilitate EMT is not well understood. In this issue of the JCI, Venkov et al. report on their examination of fibroblasts resulting from EMT and describe a novel protein-DNA complex that is essential for transcription of fibroblast-specific protein 1 (FSP1) and sufficient to induce early EMT events (see the related article beginning on page 482). Collectively, their results suggest that this complex is an important regulator of the EMT transcriptome.
\end{abstract}

During development and adult organ pathogenesis, cells are in a constant state

Nonstandard abbreviations used: $\mathrm{CBF}-\mathrm{A}, \mathrm{CArG}$ box-binding factor-A; EMT, epithelial-mesenchymal transition; FSP1, fibroblast-specific protein 1; FTS-1, fibroblast transcription site- 1 ; KAP-1, KRAB-associated protein 1.

Conflict of interest: The authors have declared that no conflict of interest exists.

Citation for this article: J. Clin. Invest. 117:304-306 (2007). doi:10.1172/JCI31200. the characteristics of mesenchymal cells. EMT is regarded as a critical regulator of metazoan embryogenesis and physiological processes such as wound healing. EMT also contributes significantly in pathologies such as tissue fibrosis and cancer metastasis. Hallmarks of EMT include: (a) the downregulation of cell adhesion molecules such as E-cadherin; (b) the increased expression of MMPs to assist in the degradation of the basement membrane; (c) the activation of the Rac/Rho/Cdc42 family small GTPase to bring about cytoskeleton rearrangement; and (d) the nuclear translocation of several transcription factors including $\beta$-catenin and the $\mathrm{T}$ cell factor/ lymphocyte enhancer factor 1 (TCF/LEF1) complex, Snail1, Snail2, and Twist $(1,2)$. The adoption of a fibroblast-like transcription profile is crucial for the survival of the cells undergoing EMT. Several key transcription factors have been described (1); however, it is now clear that more such transcriptional regulators are required to govern the complex EMT transcriptome. 